\title{
A Thrust Allocation Method for Efficient Dynamic Positioning of a Semisubmersible Drilling Rig Based on the Hybrid Optimization Algorithm
}

\author{
Luman Zhao ${ }^{1}$ and Myung-Il Roh $^{2}$ \\ ${ }^{1}$ Department of Naval Architecture and Ocean Engineering, Seoul National University, Seoul 08826, Republic of Korea \\ ${ }^{2}$ Department of Naval Architecture and Ocean Engineering, Research Institute of Marine System Engineering, \\ Seoul National University, Seoul 08826, Republic of Korea \\ Correspondence should be addressed to Myung-Il Roh; miroh@snu.ac.kr
}

Received 7 April 2015; Revised 3 August 2015; Accepted 5 August 2015

Academic Editor: Chunlin Chen

Copyright (C) 2015 L. Zhao and M.-I. Roh. This is an open access article distributed under the Creative Commons Attribution License, which permits unrestricted use, distribution, and reproduction in any medium, provided the original work is properly cited.

A thrust allocation method was proposed based on a hybrid optimization algorithm to efficiently and dynamically position a semisubmersible drilling rig. That is, the thrust allocation was optimized to produce the generalized forces and moment required while at the same time minimizing the total power consumption under the premise that forbidden zones should be taken into account. An optimization problem was mathematically formulated to provide the optimal thrust allocation by introducing the corresponding design variables, objective function, and constraints. A hybrid optimization algorithm consisting of a genetic algorithm and a sequential quadratic programming (SQP) algorithm was selected and used to solve this problem. The proposed method was evaluated by applying it to a thrust allocation problem for a semisubmersible drilling rig. The results indicate that the proposed method can be used as part of a cost-effective strategy for thrust allocation of the rig.

\section{Introduction}

Research Background. A dynamic positioning (DP) system is especially useful to deep-sea working vessel, such as drilling vessel and floating crane. The DP system can maintain the position (with a fixed location or on a predetermined track) and the heading of a working vessel exclusively through the use of active thrusters and propellers. To keep the position means to maintain the desired position within the normal excursion from the desired position and heading in the horizontal plane. For an offshore vessel, which is a semisubmersible drilling rig illustrated in Figure 1 in this case, the DP system generally consists of a position and heading reference system with sensor units, a control system, a power system, and a thruster system [1]. The position and heading reference system, along with the wind sensors and gyrocompasses, can provide the control system with information on the position of the vessel and the magnitude and direction of the environmental forces that affect the position. All of the information obtained is then used to adjust the vessel itself.

The DP system must be very responsive to changes in the conditions due to weather, water depth, and so on in order to properly control the operating parameters of the vessel. Despite the importance of the DP system to the vessel, its use in a real working environment still faces many challenges, such as a power failure and a thruster malfunction, due to its complexity. The fuel expenditure of the DP system is a considerable burden since it requires a large amount of energy. This means that the DP system is overactuated, which renders the thruster to have infinite allocation solutions. Therefore, the thrust allocation can be formulated as an optimization process under numerous constraints in order to minimize power consumption.

Related Works. In the literature, many methods have been proposed to achieve thrust allocation. Johansen and Fossen [2] provided a recent review of thrust allocation methods, 


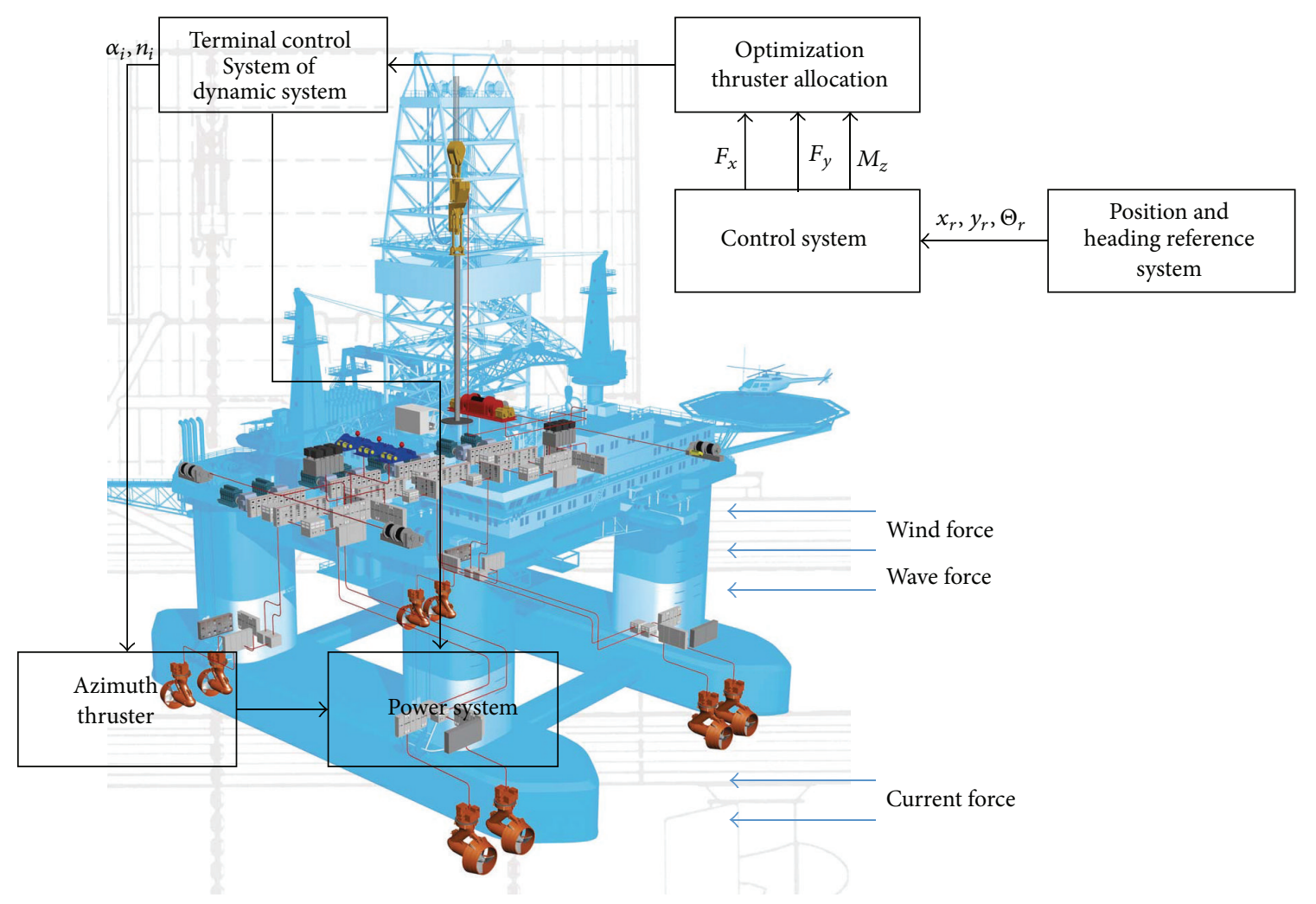

FIGURE 1: Dynamic positioning system configured for 8 thrusters of a semisubmersible drilling rig.

but most of these have focused on proposing optimization algorithms intended for thrust allocation for industries, such as automobile, aerospace [3], and robotics, and not for ocean engineering. The relationship between the power consumption and the resulting thrust can usually be approximated by using a quadratic function, so some researchers have formulated optimization problems for thrust allocation as quadratic programming problems and have solved these with some variant of quadratic programming (QP) algorithms [4].

Some of the methods intended for ocean engineering have considered cases with ships or with offshore vessels. Wit [5] proposed a method to achieve the optimal thrust allocation for a ship. The thrust and azimuth direction of the thrusters were selected as design variables for the optimization problem, and the QP algorithm, which is a local optimization algorithm, was used to solve the problem. Similarly, Liang and Cheng [6] and Johansen et al. [7] proposed methods for the ship that include a similar formulation of an optimization problem and a solution through the use of a sequential quadratic programming (SQP) algorithm. Parikshit [8] also proposed a method to achieve the optimal thrust allocation for a drilling rig. The thrust and azimuth direction of thrusters were selected as design variables of an optimization problem, and various global optimization algorithms, such as genetic algorithm (GA) and ITHS (Intelligent Tuned Harmony Search), were used to solve this problem. Zhao et al. [9] also proposed a method that includes a similar formulation of an optimization problem and solution for a drilling rig by using GA.
In this manner, most studies in the field of ocean engineering have determined the thrust and the azimuth direction of the thrusters to be design variables for an optimization problem. In fact, the thrust can be expressed as a function of the speed of rotation, the diameter, and the thrust coefficient of a thruster. In order to reflect the specifications of the given thruster and to more easily control the thruster, the speed of rotation can be used as a design variable instead of the thrust. Meanwhile, a local optimization algorithm can be used as the optimization algorithm, such as the SQP algorithm, to derive an accurate optimum. However, this method is very sensitive to a starting point for optimization and sometimes finds a local optimum. On the other hand, a global optimization algorithm, such as the GA, does not need a starting point for the optimization but can only find a rough optimum [10].

This study proposes an optimal thrust allocation method for an offshore vessel with the aim of achieving the required generalized forces and moment for dynamic positioning while at the same time minimizing total power. Finding such a method is a particularly important issue to operate an offshore vessel while using minimal energy. The method involves mathematically formulating an optimization problem for thrust allocation and selecting and using a suitable optimization algorithm to solve the problem, not developing a new algorithm. When the optimization problem is formulated, the speed of the rotation and the azimuth direction of thrusters are selected as design variables, the total power is selected as an objective function, and the required forces and moment are selected as constraints. A hybrid optimization 
TABLE 1: Summary of related studies and comparison with this study.

\begin{tabular}{lcccc}
\hline Studies & Application & Optimization algorithm & $\begin{array}{c}\text { Design variables for } \\
\text { formulation }\end{array}$ & $\begin{array}{c}\text { Consideration of } \\
\text { thruster interaction }\end{array}$ \\
\hline Wit [5] & Ship & Local algorithm (QP) & Thrust and azimuth direction & O \\
\hline $\begin{array}{l}\text { Liang and Cheng [6] } \\
\text { Johansen et al. [7] }\end{array}$ & Ship & Local algorithm (SQP) & Thrust and azimuth direction \\
\hline Parikshit [8] & Drilling rig & Global algorithm (GA, ITHS, etc.) & Thrust and azimuth direction \\
\hline Zhao et al. [9] & Drilling rig & Global algorithm (GA) & Thrust and azimuth direction & $\bigcirc$ \\
\hline This study & Drilling rig & $\begin{array}{c}\text { Global algorithm (hybrid } \\
\text { optimization algorithm) }\end{array}$ & $\begin{array}{c}\text { Rotation speed and azimuth } \\
\text { direction }\end{array}$ \\
\hline
\end{tabular}

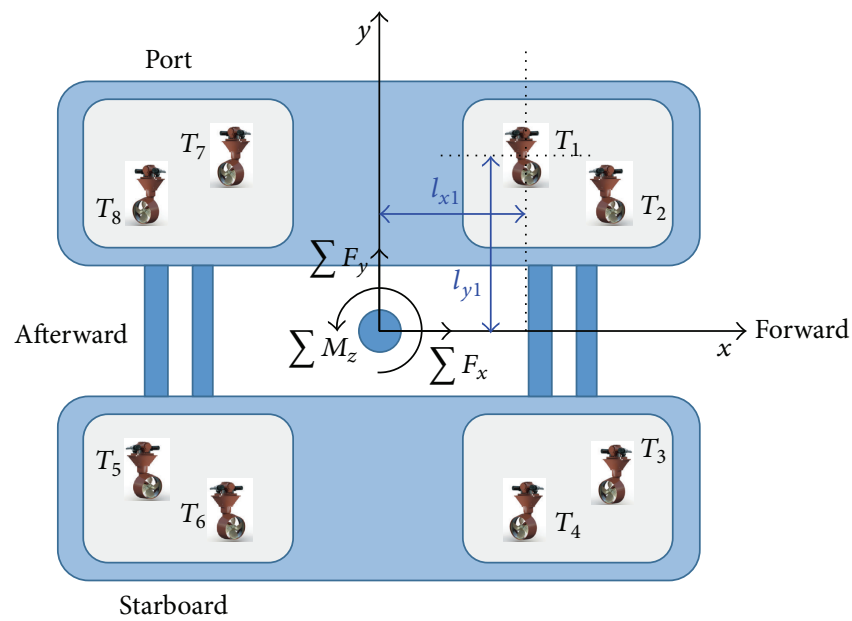

FIGURE 2: Schematic of thruster arrangement of a drilling rig with eight thrusters (Plan view).

algorithm that incorporates global and local optimization algorithms was used for the optimization.

Table 1 shows a summary of related studies and a comparison of them with this study.

\section{Thrust Allocation for Dynamic Positioning}

A thruster system is important for a drilling rig to maintain its position and heading since it can simultaneously provide transverse and longitudinal thrust. An azimuth thruster consists of an electric podded drive that is fitted to the hull and can rotate 360 degrees along the horizontal angle to provide thrust in all directions. The azimuth thruster has been selected in this study because it can provide thrust in any direction in order to act as a propulsor for dynamic positioning and target optimization of a drilling rig. However, the thruster system is usually overactuated in practice, so a thrust allocation problem can be formulated into a constrained optimization problem for the system.

Figure 2 depicts a schematic diagram for the thruster arrangement of the drilling rig and the sign conventions for the forces $\left(F_{x}\right.$ and $\left.F_{y}\right)$ and moment $\left(M_{z}\right)$. In this figure, $\sum F_{x}, \sum F_{y}$, and $\sum M_{z}$ represent the total forces required in the longitudinal $(x)$ and transverse $(y)$ directions and the total required moment about the vertical $(z)$ direction from the control system, as shown in Figure 1, respectively. Note that the positions of the thrusters are given in a $2 \mathrm{D}$ coordinate system (only the horizontal forces and moment are considered). The coordinate system has its origin at the center of gravity $(\mathrm{CoG})$ of the drilling rig.

The environmental forces and the moment tend to move the drilling rig away from its original position during operation at sea. In order to move the drilling rig back to its original or reference position, the control system in the DP system of the drilling rig should first calculate the total forces (thrusts) and moment required. At this time, thrust allocation should be conducted to determine the thrust and azimuth direction of each thruster so that the required forces (and moment) are generated in each of the longitudinal $\left(\sum F_{x}\right)$ and transverse $\left(\sum F_{y}\right)$ and vertical $\left(\sum M_{z}\right)$ directions. The relationship between the thrust and the azimuth direction of each thruster and the total forces and moment that are required can be stated as in (1) to (3). These equations are the governing equations for the thrust allocation and can be used as equality constraints in an optimization problem:

$$
\begin{aligned}
& \sum F_{x}=\left(F_{1} \cos \alpha_{1}+F_{2} \cos \alpha_{2}+\cdots+F_{n} \cos \alpha_{n}\right), \\
& \sum F_{y}=\left(F_{1} \sin \alpha_{1}+F_{2} \sin \alpha_{2}+\cdots+F_{n} \sin \alpha_{n}\right), \\
& \sum M_{z}=\left(F_{1} \cos \alpha_{1} \cdot l_{y 1}+F_{2} \cos \alpha_{2} \cdot l_{y 2}+\cdots\right. \\
& \quad+F_{n} \cos \alpha_{n} \cdot l_{y n}+F_{1} \sin \alpha_{1} \cdot l_{x 1}+F_{2} \sin \alpha_{2} \cdot l_{x 2} \\
& \left.\quad+\cdots+F_{n} \sin \alpha_{n} \cdot l_{x n}\right) .
\end{aligned}
$$


Supposing that the drilling rig is equipped with $i$ azimuth thrusters, $F_{i}$ is the thrust from $i$ th thruster and $\alpha_{i}$ is the azimuth direction (angle) of $i$ th thruster. $l_{x i}$ and $l_{y i}$ are the distances of $i$ th thruster from the CoG of the drilling rig. Equation (1) indicates that the longitudinal component of the force generated from the thrust and azimuth direction of each thruster should be equal to the total force required in the longitudinal direction. Similarly, (2) indicates that the transverse component of the force from the thrust and azimuth direction of each thruster should be equal to the total force required in the transverse direction. Equation (3) means that the vertical component of the moment generated from the thrust and azimuth direction of each thruster should be equal to the total required moment about the vertical direction.

The goal of achieving optimal thrust allocation is to guarantee efficient power use when dynamically positioning the drilling rig in the specific conditions of a given ocean environment. Meanwhile, the economic impact of minimizing power consumption is also taken into account with the ultimate aim of ensuring the stability and capability of the drilling rig during operation.

\section{Thrust Allocation Method}

3.1. Optimization Problem for Thrust Allocation. This study mathematically formulated an optimization problem for thrust allocation, and each component of the optimization problem is described below in detail.

3.1.1. Design Variables. Thrust allocation involves determining the thrust $\left(F_{i}\right)$ and azimuth direction $\left(\alpha_{i}\right)$ of each thruster to generate the forces in longitudinal and transverse directions and the moment about the vertical direction that are required for the DP system of the drilling rig. Thus, the thrust and azimuth direction of each thruster can be used as design variables in the thrust allocation optimization problem. The objective function that is commonly used for this problem is to minimize the total power of the thrusters due to energy savings. In order to use this power as the objective function, it should be mathematically formulated as a function of the design variables. The total power will be proportional to the sum of the thrusts for each thruster. Some researchers [5-9] used the thrust and the azimuth direction of each thruster as design variables and have formulated the objective function (minimization of total power of thrusters) with the corresponding design variables. However, the rotational speed $\left(n_{i}\right)$ and the azimuth direction $\left(\alpha_{i}\right)$ of each thruster can be used as design variables to reflect the characteristics of the given thruster, to more easily control the thruster, and to more delicately represent the objective function. Thus, the design variables for the thrust allocation optimization problem in this study are as follows:

$n_{i}$ : speed of rotation of each thruster.

$\alpha_{i}$ : azimuth direction of each thruster.
3.1.2. Objective Function. In this study, the speed of the rotation $\left(n_{i}\right)$ and the azimuth direction $\left(\alpha_{i}\right)$ of each of the thrusters were used as design variables, and thus the objective function (minimization of the total power for the thrusters) was mathematically formulated according to the following procedure where in fact the power consumed by the electric propulsion system depends on the speed of rotation and the azimuth direction of the thrusters.

The thrust $T$ and torque $Q$ of a thruster can be expressed as functions of the speed of rotation $(n)$ :

$$
\begin{aligned}
& T=\rho D^{4} K_{T} n^{2}, \\
& Q=\rho D^{5} K_{Q} n^{2},
\end{aligned}
$$

where $\rho$ is the density of the sea water, $D$ is the propeller diameter of the thruster, $K_{T}$ and $K_{\mathrm{Q}}$ are the thrust and torque coefficients, respectively, and $n$ is the speed of the rotation of the thruster. The expressions for $K_{T}$ and $K_{Q}$ can be obtained from the so-called open water test of the thruster. These nondimensional coefficients for thrust and torque were derived through a regression analysis that can be described according to the following parameters [11]:

$$
\begin{aligned}
& K_{T}=\sum_{s, t, u, v} C_{s, t, u, v}^{T}(J)^{s}\left(\frac{P}{D}\right)^{t}\left(\frac{A_{E}}{A_{O}}\right)^{u}(Z)^{v}, \\
& K_{Q}=\sum_{s, t, u, v} C_{s, t, u, v}^{Q}(J)^{s}\left(\frac{P}{D}\right)^{t}\left(\frac{A_{E}}{A_{O}}\right)^{u}(Z)^{v} .
\end{aligned}
$$

$C_{s, t, u, v}^{T}$ and $C_{s, t, u, v}^{\mathrm{Q}}$ coefficients and $s, t, u$, and $v$ terms can be found in a report of the Wageningen B-Series propellers [11] and were used in this study. Here, $P / D$ is the pitch diameter ratio, $A_{E} / A_{O}$ is the blade area ratio, and $Z$ is the number of blades of the thruster. The coefficient for the advance $J$ is a nondimensional description of the thruster performance that can be expressed according to the speed of rotation $n$ and the speed of the advance of the thruster defined as $V_{A}$, which can be represented by the speed near each thruster $(V)$ and the azimuth direction of each thruster $(\alpha)$. Since each of the thrusters has a different speed of rotation in practice, $K_{T}$ and $K_{\mathrm{Q}}$ of each thruster can be different:

$$
J=\frac{V_{A}}{n D}=\frac{V \cos \alpha}{n D} .
$$

Now, it is time to formulate the total power consumed by the thrusters of the drilling rig. The power consumption $(P)$ can be formulated as

$$
P=2 \pi n_{i} Q_{i}
$$

By combining (5) and (8), the power consumption of the thrusters can be rewritten as

$$
P=2 \pi \rho D^{5} \sum_{i=1}^{N} K_{\mathrm{Q}_{i}} n_{i}^{3},
$$

where $N$ is the number of thrusters. 


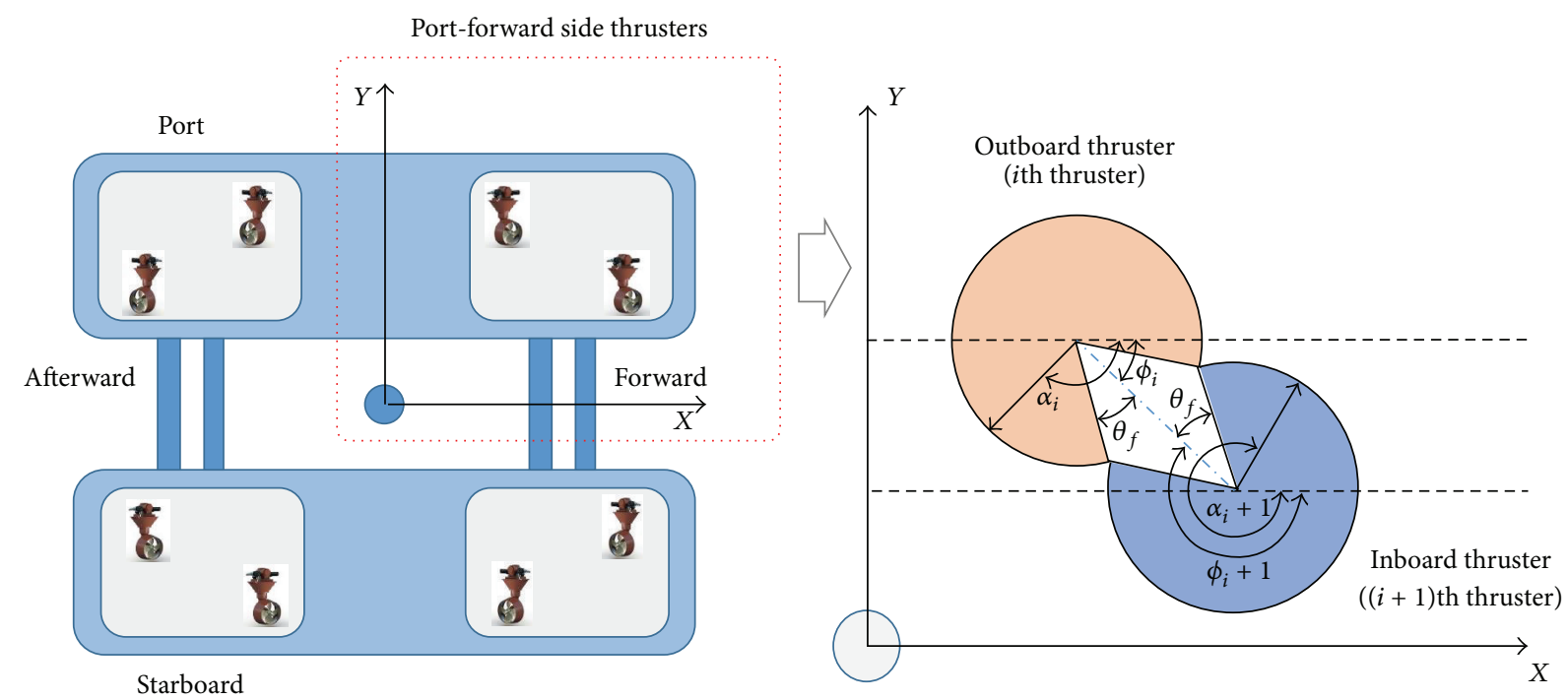

FIGURE 3: Interaction of two closely spaced azimuth thrusters.

Therefore, the objective function can be written relating the speed of rotation and the azimuth direction of each thruster. In this study, the total power (power consumption) of the thrusters is minimized by setting the objective function for the thrust allocation optimization problem as

$$
\text { Minimize } P=2 \pi \rho D^{5} \sum_{i=1}^{N} K_{Q_{i}} n_{i}^{3} \text {. }
$$

3.1.3. Constraints. To obtain a valid thrust allocation, the governing equations shown in (11) to (13) should be satisfied. The equations represent the thrust and azimuth direction of each thruster that generate the forces required in longitudinal $\left(\sum F_{x}\right)$ and transverse $\left(\sum F_{y}\right)$ directions as well as the moment required about vertical direction $\left(\sum M_{z}\right)$ to dynamically position the vessel. If these equations cannot be satisfied, the drilling rig starts to drift. Thus, these equations are used as equality constraints for the optimization problem.

The governing equation for the longitudinal force can be stated as

$$
\begin{aligned}
& \sum F_{x} \\
& \quad=\rho D^{2} K_{T_{i}}\left(n_{1}^{2} \cos \alpha_{1}+n_{2}^{2} \cos \alpha_{2}+\cdots+n_{i}^{2} \cos \alpha_{i}\right) .
\end{aligned}
$$

Similarly, the governing equation for the transverse force can be stated as

$$
\begin{aligned}
& \sum F_{y} \\
& \quad=\rho D^{2} K_{T_{i}}\left(n_{1}^{2} \sin \alpha_{1}+n_{2}^{2} \sin \alpha_{2}+\cdots+n_{i}^{2} \sin \alpha_{i}\right) .
\end{aligned}
$$

And finally the governing equation for the yawing moment can be stated as

$$
\begin{aligned}
& \sum M_{z}=\rho D^{2} K_{T_{i}}\left(n_{1}^{2} \sin \alpha_{1} l_{x_{1}}+n_{2}^{2} \sin \alpha_{2} l_{x_{2}}+\cdots\right. \\
& \quad+n_{i}^{2} \sin \alpha_{i} l_{x_{i}}+n_{1}^{2} \cos \alpha_{1} l_{y_{1}}+n_{2}^{2} \cos \alpha_{2} l_{y_{2}}+\cdots \\
& \left.\quad+n_{i}^{2} \cos \alpha_{i} l_{y_{i}}\right)
\end{aligned}
$$

where $\sum F_{x}, \sum F_{y}$, and $\sum M_{z}$ are the forces required in the longitudinal and transverse directions and the moment required about the vertical direction, respectively, and are all given from the control system in the dynamic positioning system.

It is essential to minimize the thruster interaction of a semisubmersible drilling rig to ensure effective DP operation $[5,12]$. In order to find a solution to provide the most effective thrust for operation, additional constraints should be considered by taking into account the physical locations of the thrusters. Thrusters that are close together have an influence on each other, and if one thruster is in the stream of another, the efficiency would drop significantly. To avoid having this happen, certain angles must be prohibited for the azimuth direction. Each thruster is assumed to have a forbidden angle of $\theta_{f}$ for the closest thruster in order to prevent the direct interaction of the thruster, as shown in Figure 3. With the port-forward pair, the center of the angle of the outboard thruster $i$ is $\phi_{i}$ and for the inboard thruster $(i+1)$ it is $\phi_{i+1}$. The positive angle is measured clockwise while the negative angle is measured anticlockwise from the horizontal axis. Figure 3 shows the ATRs (Attainable Thrust Regions) for the two port-forward side thrusters $i$ and $(i+1)$. 
The ATR represents a set of all physically possible surge and sway forces for $i$ th thruster.

Considering the ATR, the constraints for the azimuth direction of thrusters $i$ and $(i+1)$ can be formulated as

$$
\begin{array}{r}
\theta_{f}-\left|\alpha_{i}-\phi_{i}\right| \leq 0, \\
\theta_{f}-\left|\alpha_{i+1}-\phi_{i+1}\right| \leq 0,
\end{array}
$$

where $\theta_{f}$ represents the forbidden angle of the thrusters. $\alpha_{i}$ and $\alpha_{i+1}$ are the azimuth directions of the thrusters $i$ and $(i+1)$, respectively. $\phi_{i}$ and $\phi_{i+1}$ are the center of angle for the outboard thruster $i$ and for the inboard thruster $(i+1)$, respectively.

3.1.4. Summary. The optimization problem for thrust allocation can be summarized as follows:

$$
\begin{array}{ll}
\text { Minimize } & f(\mathbf{x})=P=2 \pi \rho D^{5} \sum_{i=1}^{n} K_{\mathrm{Q}_{i}} n_{i}^{3}, \\
\text { Subject to } & g_{1}(\mathbf{x})=\sum F_{x}-\rho D^{2} K_{T_{i}}\left(n_{1}^{2} \cos \alpha_{1}+n_{2}^{2} \cos \alpha_{2}+\cdots+n_{i}^{2} \cos \alpha_{i}\right)=0, \\
& g_{2}(\mathbf{x})=\sum F_{y}-\rho D^{2} K_{T_{i}}\left(n_{1}^{2} \sin \alpha_{1}+n_{2}^{2} \sin \alpha_{2}+\cdots+n_{i}^{2} \sin \alpha_{i}\right)=0, \\
& g_{3}(\mathbf{x}) \\
& =\sum M_{z}-\rho D^{2} K_{T_{i}}\left(n_{1}^{2} \sin \alpha_{1} l_{x_{1}}+n_{2}^{2} \sin \alpha_{2} l_{x_{2}}+\cdots+n_{i}^{2} \sin \alpha_{i} l_{x_{i}}+n_{1}^{2} \cos \alpha_{1} l_{y_{1}}+n_{2}^{2} \cos \alpha_{2} l_{y_{2}}+\cdots+n_{i}^{2} \cos \alpha_{i} l_{y_{i}}\right) \\
& =0, \\
& g_{4+i}(\mathbf{x})=\theta_{f}-\left|\alpha_{i}-\phi_{i}\right| \leq 0,
\end{array}
$$

where $\mathbf{x}=\left\{n_{i}, \alpha_{i}\right\}, i=1, \ldots, N$ (N: number of thrusters).

Thus, this problem has one objective function, three equality constraints, and $N$ inequality constraints.

\subsection{Hybrid Optimization Algorithm for Thrust Allocation}

3.2.1. Overview. The optimization algorithms are generally divided into two categories: global and local. Several classes of global optimization algorithms are now available, including the genetic algorithm (GA) $[13,14]$ and the simulated annealing method. These algorithms are suitable for large-scale problems with many local optima. However, these algorithms require further iteration to obtain an acceptable optimum and not an accurate optimum, in contrast to the local optimization algorithms. Several classes of the local optimization algorithms also exist, including sequential linear programming (SLP) [15] and sequential quadratic programming (SQP) [15] and the method of feasible directions (MFD) [16]. Each of these algorithms can effectively determine an accurate optimum. However, in some cases, these algorithms find the local optimum that is closest to the given starting point.

Various attempts have been made to combine global and local optimization algorithms in order to overcome the challenges of using them separately [10, 17-21]. Most of these studies used a combination of a global (e.g., GA) and a local optimization algorithm, which can be referred to as a hybrid optimization algorithm. In this study, the hybrid optimization algorithm was selected and used by incorporating the GA and the SQP algorithm to solve an optimization problem for thrust allocation, as described in Section 3.1. In this case, the SQP algorithm was used to improve the acceptable, global optimum obtained from the GA. That is, at the end of the GA, the SQP algorithm was executed with a starting point that corresponds to the optimum obtained from the GA. Figure 4 shows the general procedure of the hybrid optimization algorithm used in this study.

An experiment on the mathematical optimization problem was performed in order to verify the efficiency, accuracy, and applicability of the hybrid optimization algorithm that was used to optimize the thrust allocation in this study. The selected problem, which is Rastrigin's problem, one of the benchmark problems, is being widely used to check the efficiency of optimization algorithms [22]. More details about comparative test of the hybrid optimization algorithm can be found in [10].

3.2.2. Optimization Procedure. The following optimization procedure was established to solve the optimization problem that was presented formulated above. First, the initial values were assumed for the design variables. At this time, the values can be randomly generated or can be manually set or extracted from an existing design. Now, these values are transferred to the hybrid optimization algorithm, and the values of an objective function and constraints are then calculated. We then check whether the current values of the design variables are at an optimum or not. If yes, the optimization process finishes and the result will be shown, and if not, the above steps will be repeated until the optimum 


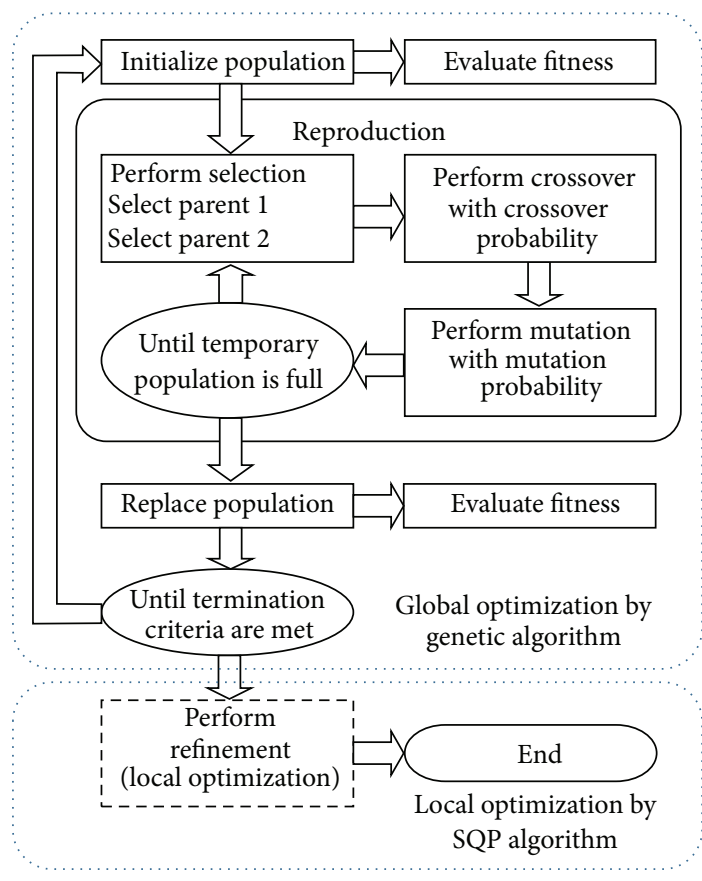

FIGURE 4: General procedure of the hybrid optimization algorithm used in this study.

is found. Figure 5 shows this optimization procedure to find an optimal thrust allocation.

\section{Application of the Thrust Allocation Method}

4.1. Optimization Target. The optimization target for this study is a semisubmersible drilling rig equipped with eight azimuth thrusters. Figure 5 shows the schematics of this drilling rig with a thruster system and its position. We assumed that each thruster has 4 propeller blades $(Z)$, a propeller diameter $(D)$ of $3.6 \mathrm{~m}$, a pitch diameter ratio $(P / D)$ of 1.1 , a blade area ratio $\left(A_{E} / A_{O}\right)$ of 0.7 , and a maximum thrust of $600 \mathrm{KN}$. In addition, we assumed that the speed $(V)$ near each thruster was of $1 \mathrm{~m} / \mathrm{s}$. The longitudinal and transverse positions of each thruster can be seen in Figure 6 .

4.2. Mathematical Formulation. This drilling rig has eight thrusters. Thus, the design variables in this problem are as follows:

$$
\begin{aligned}
\mathbf{x}= & \left\{n_{1}, \alpha_{1}, n_{2}, \alpha_{2}, n_{3}, \alpha_{3}, n_{4}, \alpha_{4}, n_{5}, \alpha_{5}, n_{6}, \alpha_{6}, n_{7}, \alpha_{7},\right. \\
& \left.n_{8}, \alpha_{8}\right\},
\end{aligned}
$$

where $n_{i}$ and $\alpha_{i}$ are the speed of rotation and the azimuth direction of $i$ th thruster, respectively, and thus this problem has 16 design variables.

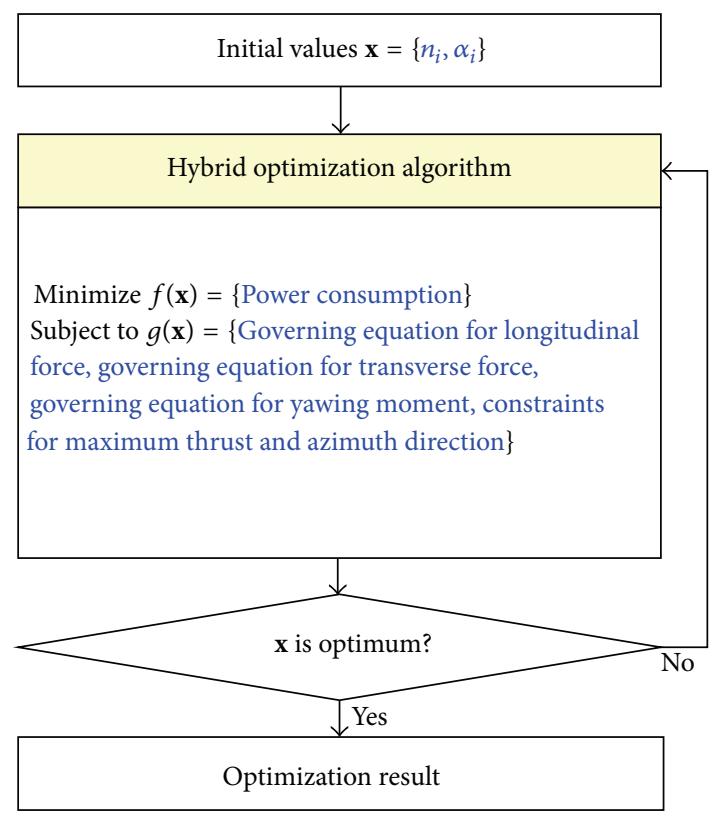

FIGURE 5: Optimization procedure for finding optimal thrust allocation.

Now, the objective function of this problem can be stated by using (10):

$$
\begin{aligned}
& \text { Minimize } f(\mathbf{x})=P=2 \pi \rho D^{5} \sum_{i=1}^{8} K_{\mathrm{Q}_{i}} n_{i}^{3} \\
& \quad=2 \pi \rho D^{5}\left(K_{\mathrm{Q}_{1}} n_{1}^{3}+K_{\mathrm{Q}_{2}} n_{2}^{3}+K_{\mathrm{Q}_{3}} n_{3}^{3}+K_{\mathrm{Q}_{4}} n_{4}^{3}\right. \\
& \left.+K_{\mathrm{Q}_{5}} n_{5}^{3}+K_{\mathrm{Q}_{6}} n_{6}^{3}+K_{\mathrm{Q}_{7}} n_{7}^{3}+K_{\mathrm{Q}_{8}} n_{8}^{3}\right),
\end{aligned}
$$

where $\rho$ is the density of sea water, $D$ is the propeller diameter of the thruster, and $K_{T}$ and $K_{Q}$ are the thruster and torque coefficients, respectively. $K_{T}$ and $K_{Q}$ were calculated from (6) by referring to the report on the Wageningen B-Series propellers [11].

The constraints for the required forces and moment can be stated using (11) to (13):

$$
\begin{aligned}
& g_{1}(\mathbf{x})=\sum F_{x}-\rho D^{2}\left(K_{T_{1}} n_{1}^{2} \cos \alpha_{1}+K_{T_{2}} n_{2}^{2} \cos \alpha_{2}\right. \\
& +K_{T_{3}} n_{3}^{2} \cos \alpha_{3}+K_{T_{4}} n_{4}^{2} \cos \alpha_{4}+K_{T_{5}} n_{5}^{2} \cos \alpha_{5} \\
& \left.+K_{T_{6}} n_{6}^{2} \cos \alpha_{6}+K_{T_{7}} n_{7}^{2} \cos \alpha_{7}+K_{T_{8}} n_{8}^{2} \cos \alpha_{8}\right) \\
& =0, \\
& g_{2}(\mathbf{x})=\sum F_{y}-\rho D^{2}\left(K_{T_{1}} n_{1}^{2} \sin \alpha_{1}+K_{T_{2}} n_{2}^{2} \sin \alpha_{2}\right. \\
& \quad+K_{T_{3}} n_{3}^{2} \sin \alpha_{3}+K_{T_{4}} n_{4}^{2} \sin \alpha_{4}+K_{T_{5}} n_{5}^{2} \sin \alpha_{5} \\
& \left.+K_{T_{6}} n_{6}^{2} \sin \alpha_{6}+K_{T_{7}} n_{7}^{2} \sin \alpha_{7}+K_{T_{8}} n_{8}^{2} \sin \alpha_{8}\right)=0,
\end{aligned}
$$




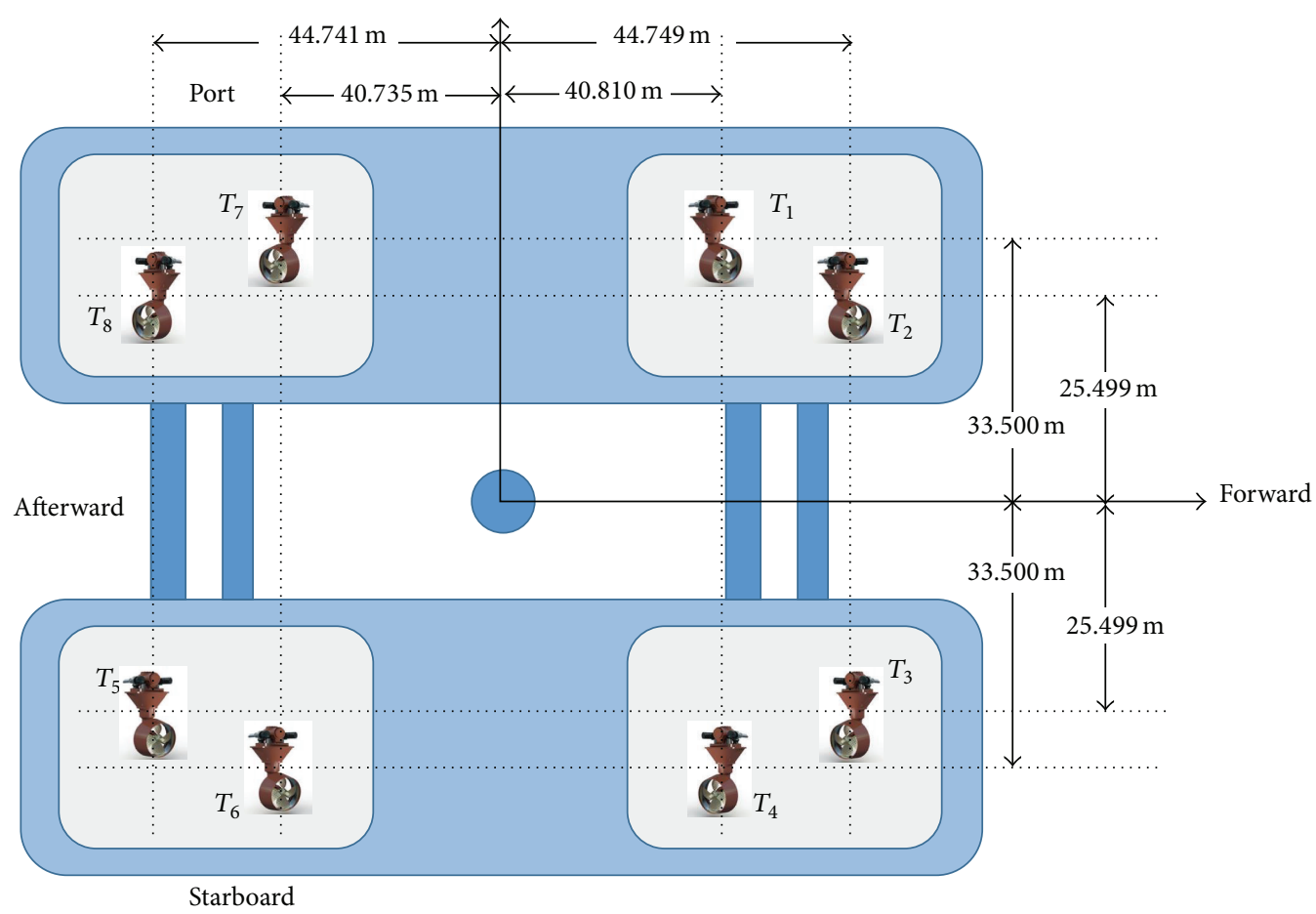

FIGURE 6: The longitudinal and transverse positions of eight azimuth thrusters (Plan view).

$$
\begin{aligned}
& g_{3}(\mathbf{x})=\sum M_{z}-\rho D^{2}\left(K_{T_{1}} n_{1}^{2} \sin \alpha_{1} l_{x_{1}}\right. \\
& +K_{T_{2}} n_{2}^{2} \sin \alpha_{2} l_{x_{2}}+K_{T_{3}} n_{3}^{2} \sin \alpha_{3} l_{x_{3}} \\
& +K_{T_{4}} n_{4}^{2} \sin \alpha_{4} l_{x_{4}}+K_{T_{5}} n_{5}^{2} \sin \alpha_{5} l_{x_{5}} \\
& +K_{T_{6}} n_{6}^{2} \sin \alpha_{6} l_{x_{6}}+K_{T_{7}} n_{7}^{2} \sin \alpha_{7} l_{x_{7}} \\
& +K_{T_{8}} n_{8}^{2} \sin \alpha_{8} l_{x_{8}}+K_{T_{1}} n_{1}^{2} \cos \alpha_{1} l_{y_{1}} \\
& +K_{T_{2}} n_{2}^{2} \cos \alpha_{2} l_{y_{2}}+K_{T_{3}} n_{3}^{2} \cos \alpha_{3} l_{y_{3}} \\
& +K_{T_{4}} n_{4}^{2} \cos \alpha_{4} l_{y_{4}}+K_{T_{5}} n_{5}^{2} \cos \alpha_{5} l_{y_{5}} \\
& +K_{T_{6}} n_{6}^{2} \cos \alpha_{6} l_{y_{6}}+K_{T_{7}} n_{7}^{2} \cos \alpha_{7} l_{y_{7}} \\
& \left.+K_{T_{8}} n_{8}^{2} \cos \alpha_{8} l_{y_{8}}\right)=0 .
\end{aligned}
$$

Equations (18) to (20) ensure that the forces generalized by the eight thrusters are equal to the forces demanded from the control system, and the moment should also be satisfied in the same way to keep the drilling rig in the desired position.

In order to overcome the energy loss due to the thrusterthruster interaction, the ATR should be considered, excluding forbidden zones, by using (14), as shown in Figure 7. For this, each thruster should satisfy the following equations simultaneously:

$$
\begin{aligned}
& g_{4}(\mathbf{x})=25^{\circ}-\left|\alpha_{1}-63.44^{\circ}\right| \leq 0, \\
& g_{5}(\mathbf{x})=25^{\circ}-\left|\alpha_{2}+116.56^{\circ}\right| \leq 0, \\
& g_{6}(\mathbf{x})=25^{\circ}-\left|\alpha_{3}-116.56^{\circ}\right| \leq 0,
\end{aligned}
$$

$$
\begin{aligned}
& g_{7}(\mathbf{x})=25^{\circ}-\left|\alpha_{4}+63.44^{\circ}\right| \leq 0, \\
& g_{8}(\mathbf{x})=25^{\circ}-\left|\alpha_{5}-63.44^{\circ}\right| \leq 0, \\
& g_{9}(\mathbf{x})=25^{\circ}-\left|\alpha_{6}+116.56^{\circ}\right| \leq 0, \\
& g_{10}(\mathbf{x})=25^{\circ}-\left|\alpha_{7}-116.56^{\circ}\right| \leq 0, \\
& g_{11}(\mathbf{x})=25^{\circ}-\left|\alpha_{8}+63.44^{\circ}\right| \leq 0 .
\end{aligned}
$$

In addition, there are some limitations on the maximum thrust and azimuth direction for each thruster. Thus, such limitations were also considered as additional constraints in this study. The additional constraints about the maximum thrust and the azimuth direction of each thruster can be stated in the following equations, respectively:

$$
\begin{aligned}
& g_{11+i}=T_{i}-600(\mathrm{KN}) \leq 0, \\
& g_{19+i}=\left|\alpha_{i}\right|-180^{\circ} \leq 0,
\end{aligned}
$$

where $i=1, \ldots, 8$.

Thus, this problem has 3 equality constraints and 24 inequality constraints.

4.3. Optimization Result. The problem in (16) to (23) was solved by using the hybrid optimization algorithm described in Section 3.2, and then the problem was also solved by using the GA in order to compare their relative efficiencies. We assumed that the total required forces $\left(\sum F_{x}, \sum F_{y}\right)$ and moment $\left(\sum M_{z}\right)$ are given from the control system at 14 time steps, as stated in [8] and as shown in Table 2. For example, 


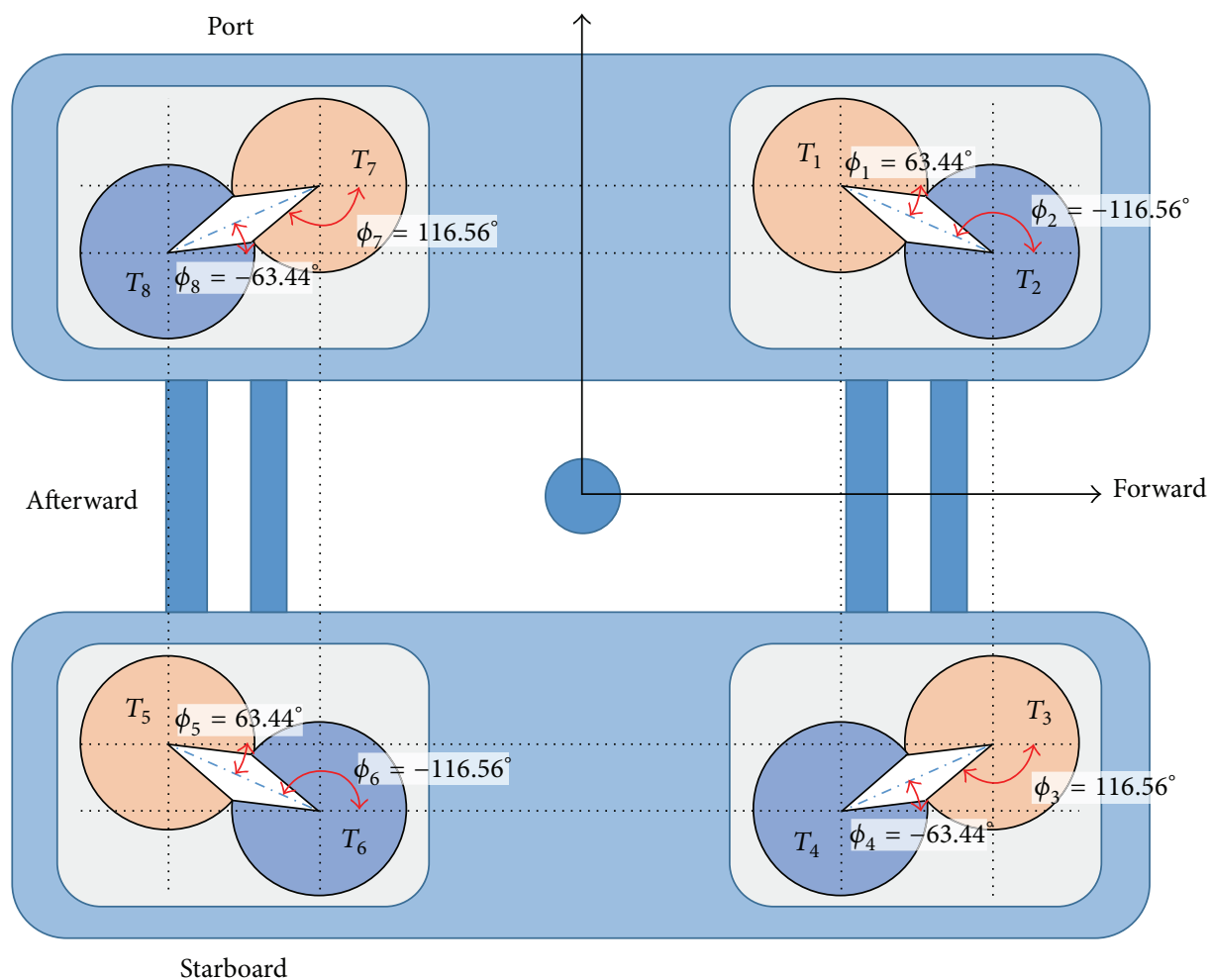

FIgURE 7: Schematic diagram of thruster interaction layout (Plan view).

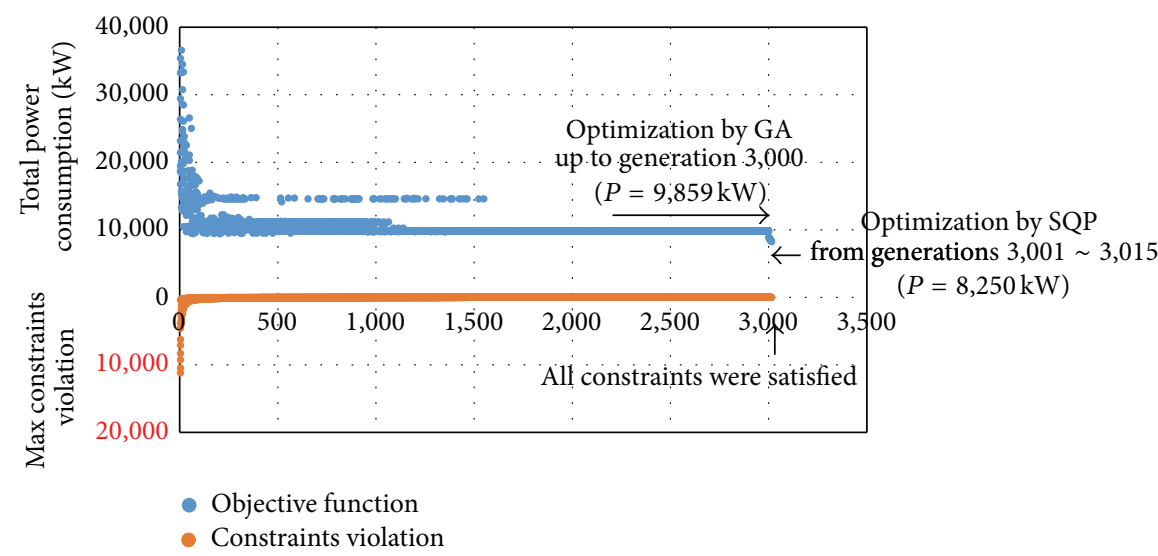

FIGURE 8: Convergence history of the optimization by the hybrid optimization algorithm for first time step.

the values for the first time step are $50 \mathrm{KN},-600 \mathrm{KN}$, and $-64,000 \mathrm{KN} \cdot \mathrm{m}$. For a total of 14 inputs for the total required forces and moment, the optimization was performed by using the GA and the hybrid optimization algorithm, and the optimization results are shown in Table 2. As mentioned earlier, the hybrid optimization algorithm is a combination of the GA and the SQP algorithm. That is, at the end of the GA, the SQP algorithm was executed with a starting point that corresponds to the optimum obtained from the GA. With respect to the parameters for the GA, the number of individuals in the population was 200 , the maximum number of generations was 3,000 , the crossover probability was 0.8 , and the mutation probability was 0.01 [23]. As shown in Table 2, the hybrid optimization algorithm outperforms the GA although the GA yielded similar results for certain time steps. Figure 8 shows a convergence history of the optimization results for the first time step. This figure shows that the objective function is minimized and that the constraints are satisfied at the same time.

To evaluate the applicability of the proposed method, the optimization results of this study were compared against those obtained in Parikshit's study [8]. The same inputs were used for the total forces and moment required from the control system for the comparative test. Parikshit used an ITHS (Intelligent Tuned Harmony Search) algorithm to solve this problem. The ITHS algorithm maintains a proper balance 


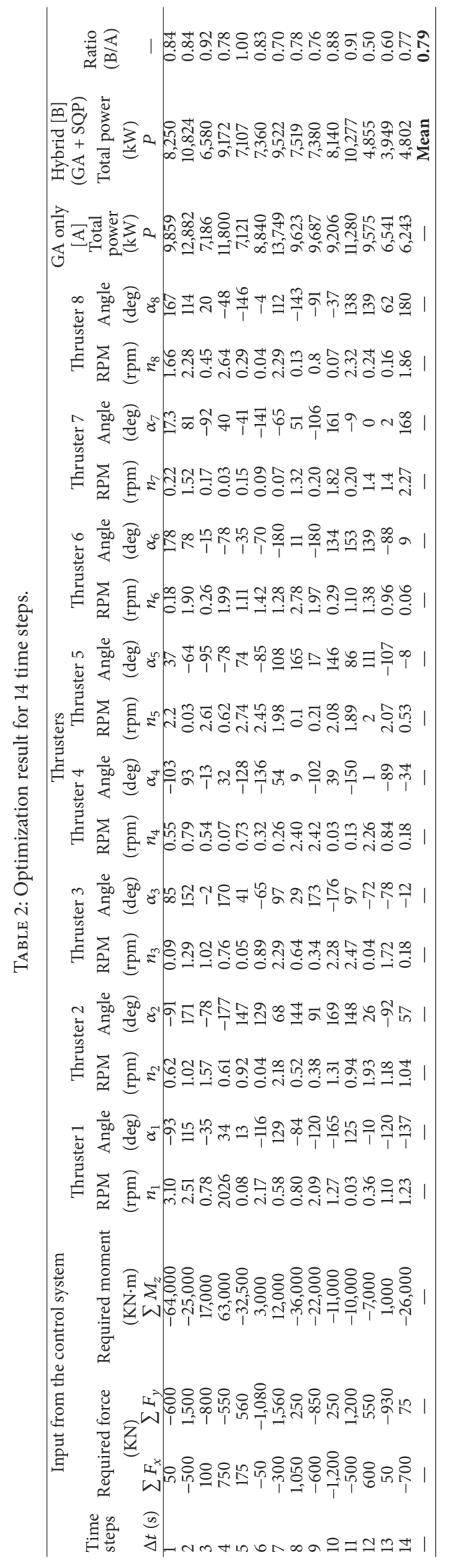


TABLE 3: Comparison of the optimization results between the existing study and this study.

\begin{tabular}{lccc}
\hline Time steps & $\begin{array}{c}\text { ITHS }[\mathrm{A}] \\
\text { Total power }(\mathrm{kW})\end{array}$ & $\begin{array}{c}\text { Hybrid }[\mathrm{B}] \\
\text { Total power }(\mathrm{kW})\end{array}$ & $\begin{array}{c}\text { Ratio } \\
(\mathrm{B} / \mathrm{A})\end{array}$ \\
\hline$t(\mathrm{~s})$ & $P$ & 8,250 & - \\
\hline 1 & 7,500 & 10,824 & 1.10 \\
2 & 10,850 & 6,580 & 0.99 \\
3 & 7,100 & 9,172 & 0.92 \\
4 & 7,800 & 7,107 & 1.17 \\
5 & 7,900 & 7,360 & 0.89 \\
6 & 5,800 & 9,522 & 1.26 \\
7 & 9,800 & 7,519 & 0.97 \\
8 & 7,000 & 7,379 & 1.07 \\
9 & 7,500 & 9,206 & 0.98 \\
10 & 9,800 & 10,277 & 0.83 \\
11 & 8,900 & 4,855 & 1.15 \\
12 & 6,200 & 3,949 & 0.78 \\
13 & 4,000 & 4,802 & 0.98 \\
14 & 7,600 & Mean & 0.63 \\
- & - & & $\mathbf{0 . 9 8}$ \\
\hline
\end{tabular}

between the diversification and intensification throughout the search process by automatically selecting the proper pitch adjustment strategy based on its harmony memory. Table 3 shows a comparison of the optimization results between the existing study and this study. As shown in this table, the results of this study were slightly better than those of Parikshit's study in terms of a mean value of the ratio.

\section{Conclusions and Future Works}

In this study, a thrust allocation method was proposed for a semisubmersible drilling rig in order to produce the generalized forces and moment that are required to dynamically position the rig while at the same time minimizing the total power consumed. First, a thrust allocation optimization problem was mathematically formulated with the corresponding design variables, objective function, and constraints. In terms of the design variables, the speed of rotation and the azimuth direction of each thruster were selected. As compared with some studies about optimal thruster allocation, the selection of the design variables can not only represent the objection function more precisely but also control the thruster more easily. The objective function aimed to minimize the total power of the thrusters, and, in terms of the constraints, the governing equations for the thrust and azimuth direction of each thruster were used to generate the forces required in longitudinal and transverse directions as well as the moment about the vertical direction to dynamically position the vessel. Some limitations were also used for each thruster, and additional constraints were introduced by considering the energy loss due to the thrusterthruster interaction and the maximum thrust and azimuth direction of each thruster. The hybrid optimization algorithm was then used to solve the formulated problem. Finally, the proposed method was applied to an example to find the optimal thrust allocation for the semisubmersible drilling rig with 8 thrusters. A comparative test was also performed as part of the current study. In this comparative test, the proposed method was observed to produce slightly better results (about 2\% in terms of total power) for thrust allocation than existing study, and thus the proposed method could be used to better determine a strategy to allocate the thruster of the drilling rig.

In the future, the total forces and moment required from the control system, which was the input of this study, will be estimated by considering the current position and the equations of motion of the drilling rig. That is, a more general method to dynamically position the rig will be further studied. In addition, we will improve the present hybrid optimization method and apply the improved method to the formulated problem in the future.

\section{Conflict of Interests}

The authors declare that there is no conflict of interests regarding the publication of this paper.

\section{Acknowledgments}

This work was partially supported by (a) Brain Korea 21 Plus Program (Education and Research Center for Creative Offshore Plant Engineers of Seoul National University) funded by the Ministry of Education, Republic of Korea, (b) Engineering Research Institute of Seoul National University, Republic of Korea, and (c) Research Institute of Marine Systems Engineering of Seoul National University, Republic of Korea.

\section{References}

[1] A. J. Sørensen, "A survey of dynamic positioning control systems," Annual Reviews in Control, vol. 35, no. 1, pp. 123-136, 2011.

[2] T. A. Johansen and T. I. Fossen, "Control allocation-a survey," Automatica, vol. 49, no. 5, pp. 1087-1103, 2013.

[3] C. Y. Duan, S. J. Zhang, Y. F. Zhao, and X. R. Kong, "Robust control allocation among overactuated spacecraft thrusters under ellipsoidal uncertainty," Abstract and Applied Analysis, vol. 2014, Article ID 950127, 12 pages, 2014.

[4] O. Härkegård, "Dynamic control allocation using constrained quadratic programming," Journal of Guidance, Control, and Dynamics, vol. 27, no. 6, pp. 1028-1034, 2004.

[5] C. D. Wit, Optimal thrust allocation methods for dynamic positioning of ships [M.S. thesis], Delft University of Technology, Delft, The Netherlands, 2009.

[6] C. C. Liang and W. H. Cheng, "The optimum control of thruster system for dynamically positioned vessels," Ocean Engineering, vol. 31, no. 1, pp. 97-110, 2004.

[7] T. A. Johansen, T. I. Fossen, and S. P. Berge, "Constrained nonlinear control allocation with singularity avoidance using sequential quadratic programming," IEEE Transactions on Control Systems Technology, vol. 12, no. 1, pp. 211-216, 2004. 
[8] Y. Parikshit, Analysis, design and optimization of offshore power system network [Ph.D. thesis], National University of Singapore, Singapore, 2013.

[9] D.-W. Zhao, F.-G. Ding, J.-F. Tan, Y.-Q. Liu, and X.-Q. Bian, "Optimal thrust allocation based GA for dynamic positioning ship," in Proceedings of the IEEE International Conference on Mechatronics and Automation (ICMA '10), pp. 1254-1258, Xian, China, August 2010.

[10] K.-Y. Lee, S. Cho, and M.-I. Roh, "An efficient global-local hybrid optimization method using design sensitivity analysis," International Journal of Vehicle Design, vol. 28, no. 4, pp. 300317, 2002.

[11] M. M. Bernitsas, D. Ray, and P. Kinley, "KT, KQ, and efficiency curves for the Wageningen B-series propellers," Tech. Rep., University of Michigan, Ann Arbor, Mich, USA, 1981.

[12] D. T. Brown and L. Ekstrom, "Vessel thruster-thruster interactions during azimuthing operations," in Proceedings of the 24th International Conference on Offshore Mechanics and Arctic Engineering, pp. 991-996, Halkidiki, Greece, June 2005.

[13] D. E. Goldberg, Genetic Algorithms in Search, Optimization, and Machine Learning, Addison-Wesley, Reading, Mass, USA, 1989.

[14] L. Davis, Handbook of Genetic Algorithms, Van NostrandReinhold, 1991.

[15] J. S. Arora, Introduction to Optimum Design, Elsevier, 3rd edition, 2012.

[16] G. Vanderplaats, Numerical Optimization Techniques for Engineering Design, McGraw-Hill, 1984.

[17] C. Stork and T. Kusuma, "Hybrid genetic autostatics: new approach for large-amplitude statics with noisy data," in Proceedings of the 62nd Anniversary International Meeting, pp. 11271131, The Society of Exploration Geophysicists, New Orleans, La, USA, 1992.

[18] M. J. Porsani, P. L. Stoffa, M. K. Sen, R. K. Chunduru, and W. T. Wood, "A combined genetic and linear inversion algorithm for waveform inversion," in Proceedings of the 63rd Anniversary International Meeting, pp. 692-695, The Society of Exploration Geophysicists, Washington, DC, USA, 1993.

[19] K. Albarado, R. Hartfield, W. Hurston, and R. Jenkins, "Solid rocket motor design using hybrid optimization," International Journal of Aerospace Engineering, vol. 2012, Article ID 987402, 9 pages, 2012.

[20] E. Deniz Ülker, A. Haydar, and K. Dimililer, "Application of hybrid optimization algorithm in the synthesis of linear antenna array," Mathematical Problems in Engineering, vol. 2014, Article ID 686730, 7 pages, 2014.

[21] F.-Z. Oujebbour, A. Habbal, R. Ellaia, and Z. Zhao, "Multicriteria shape design of a sheet contour in stamping," Journal of Computational Design and Engineering, vol. 1, no. 3, pp. 187-193, 2014.

[22] H. Willi and S. Klaus, Test Examples for Nonlinear Programming Codes, Springer, Berlin, Germany, 1981.

[23] J. J. Grefenstette, "Optimization of control parameters for genetic algorithms," IEEE Transactions on Systems, Man and Cybernetics, vol. 16, no. 1, pp. 122-128, 1986. 


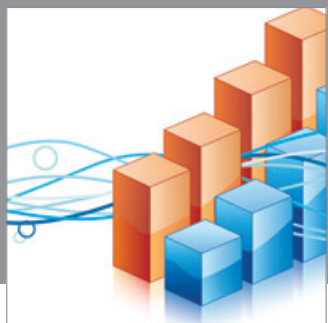

Advances in

Operations Research

mansans

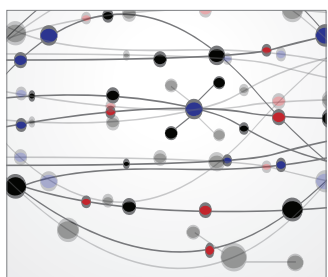

The Scientific World Journal
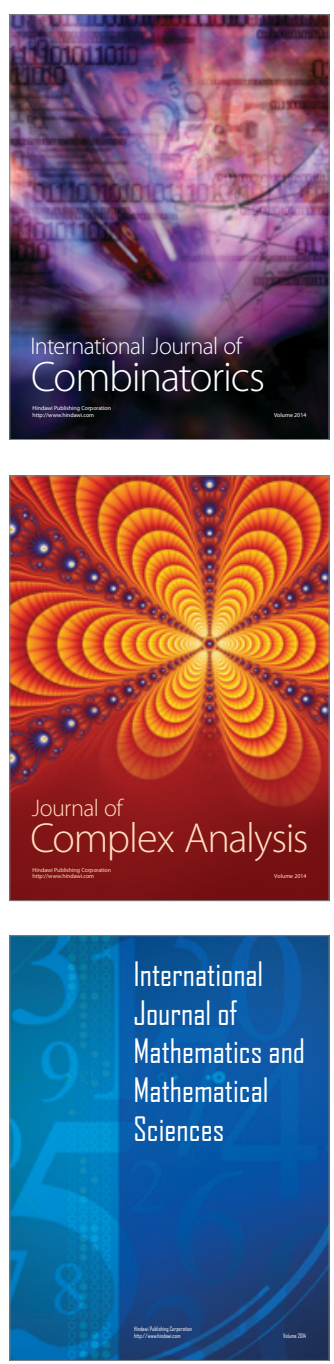
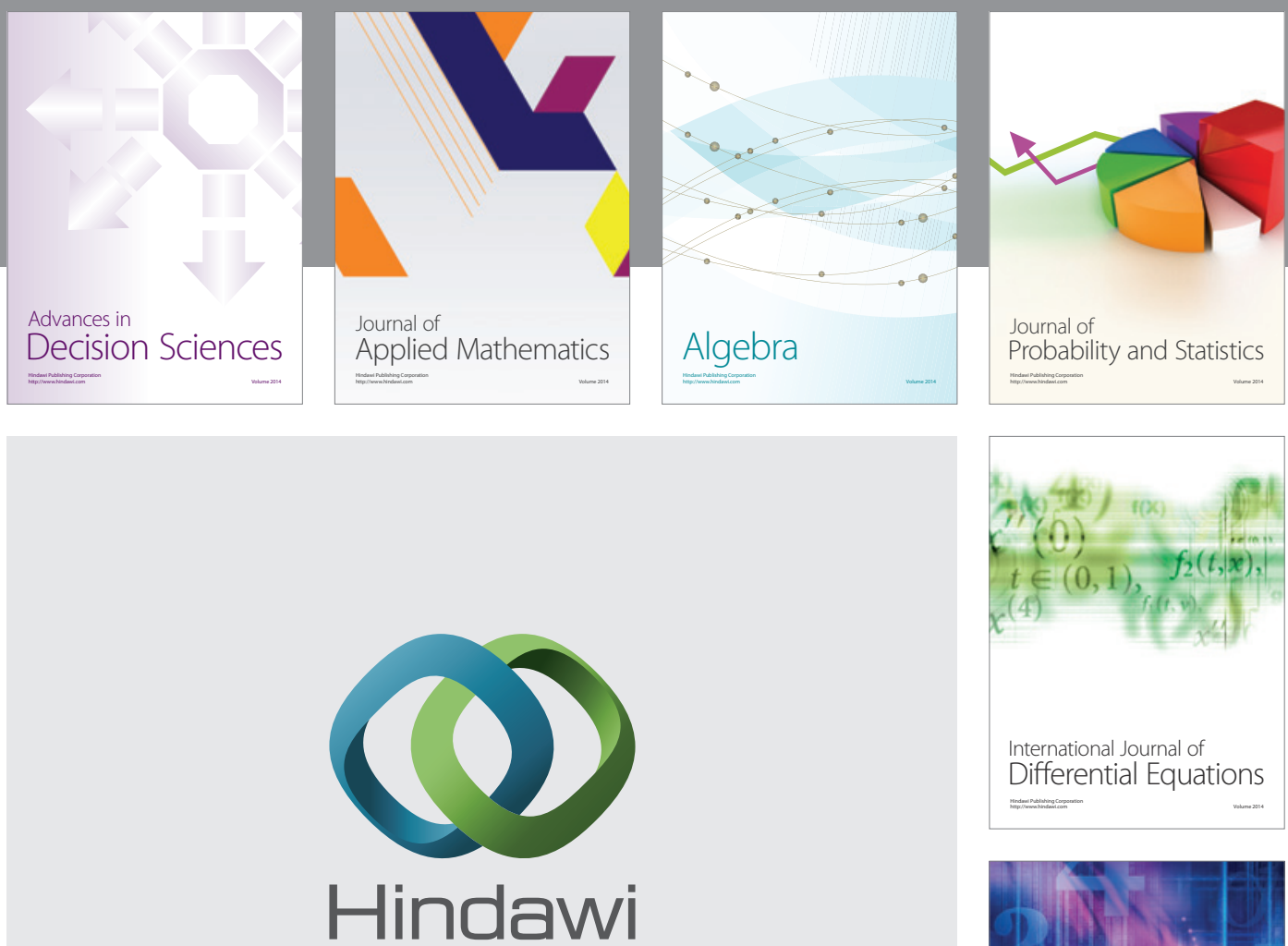

Submit your manuscripts at http://www.hindawi.com
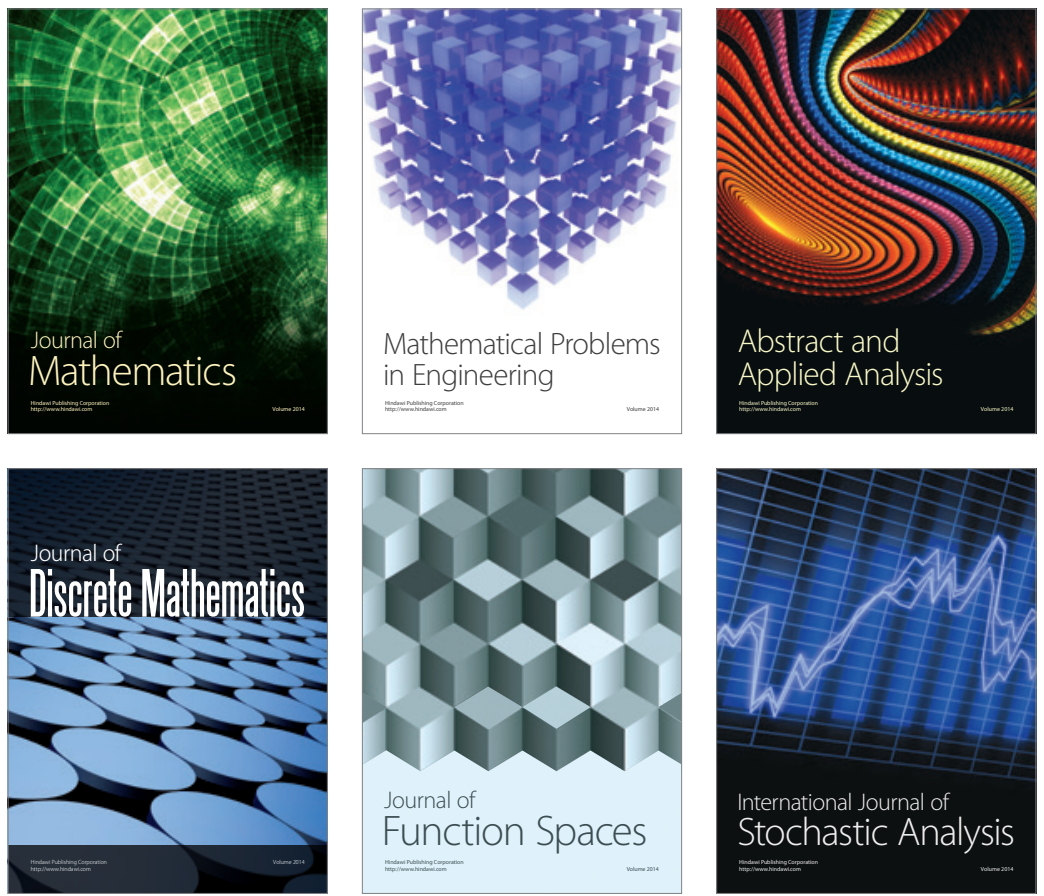

Journal of

Function Spaces

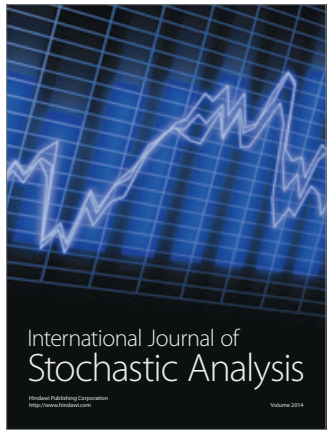

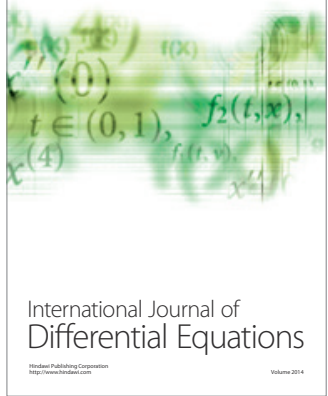
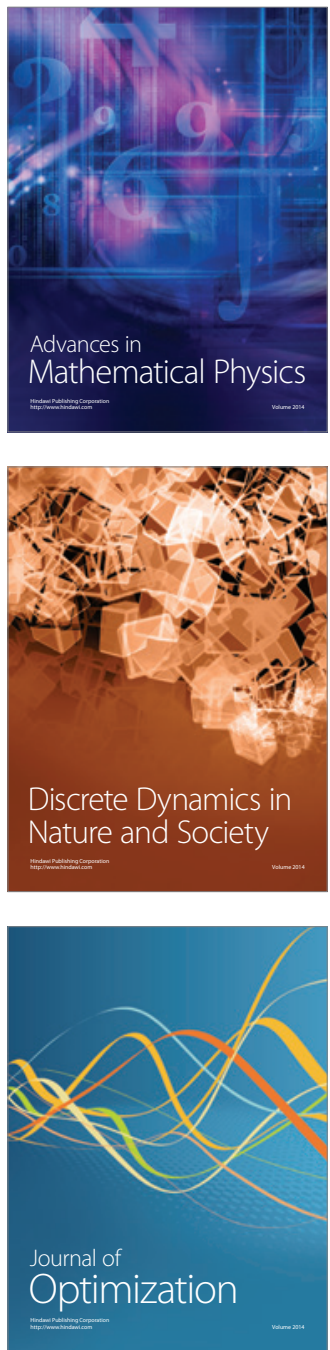